\title{
Probing the Emission States of PSR J1107-5907
}

\author{
Jingbo Wang ${ }^{1,2,3}$ (D) , George Hobbs ${ }^{4,5}$, Matthew Kerr ${ }^{6}$ (D), Ryan Shannon ${ }^{7,8}$, Shi Dai ${ }^{4}$ (D), Vikram Ravi ${ }^{9}$ (D) , Andrew Cameron ${ }^{4,5}$, \\ Jane F. Kaczmarek ${ }^{4}$, Robert Hollow ${ }^{4}$, Di Li ${ }^{5,10}$, Lei Zhang ${ }^{4,5}$, Chenchen Miao ${ }^{5}$, Mao Yuan ${ }^{5}$, Shen Wang ${ }^{5,11}$, Songbo Zhang ${ }^{4,12}$, \\ Heng $\mathrm{Xu}^{5,13,14}$, and Renxin $\mathrm{Xu}^{13,14}$ \\ ${ }^{1}$ Xinjiang Astronomical Observatory, Chinese Academy of Sciences, 150 Science 1-Street, Urumqi, Xinjiang 830011, People's Republic of China; \\ wangjingbo@xao.ac.cn \\ ${ }^{2}$ Key Laboratory of Radio Astronomy, Chinese Academy of Sciences, 150 Science 1-Street, Urumqi, Xinjiang, 830011, People's Republic of China \\ ${ }^{3}$ Xinjiang Key Laboratory of Radio Astrophysics, 150 Science 1-Street, Urumqi, Xinjiang, 830011, People's Republic of China \\ ${ }^{4}$ CSIRO Astronomy and Space Science, PO Box 76, Epping, NSW 1710, Australia \\ ${ }^{5}$ CAS Key Laboratory of FAST, National Astronomical Observatories, Chinese Academy of Sciences, Beijing 100012, People's Republic of China \\ ${ }^{6}$ Naval Research Laboratory, 4555 Overlook Ave., SW, Washington, DC 20375, USA \\ ${ }^{7}$ Centre for Astrophysics and Supercomputing, Swinburne University of Technology, P.O. Box 218, Hawthorn, Victoria 3122, Australia \\ 8 ARC Centre of Excellence for Gravitational Wave Discovery (OzGrav), Hawthorn, Victoria, Australia \\ ${ }^{9}$ Cahill Center for Astronomy and Astrophysics, MC 249-17, California Institute of Technology, Pasadena, CA 91125, USA \\ ${ }^{10}$ NAOC-UKZN Computational Astrophysics Centre, University of KwaZulu-Natal, Durban 4000, South Africa \\ ${ }^{11}$ Astronomy Department, Cornell University, Ithaca, NY 14853, USA \\ ${ }^{12}$ Purple Mountain Observatory, Chinese Academy of Sciences, Nanjing 210008, People's Republic of China \\ ${ }^{13}$ Kavli Institute for Astronomy and Astrophysics, Peking University, Beijing 100871, People's Republic of China \\ ${ }^{14}$ Department of Astronomy, School of Physics, Peking University, Beijing 100871, People's Republic of China \\ Received 2019 September 26; revised 2019 November 24; accepted 2019 November 26; published 2020 January 20
}

\begin{abstract}
The emission from PSR J1107-5907 is erratic. Sometimes the radio pulse is undetectable, at other times the pulsed emission is weak, and for short durations the emission can be very bright. In order to improve our understanding of these state changes, we have identified archival data sets from the Parkes radio telescope in which the bright emission is present, and find that the emission never switches from the bright state to the weak state, but instead always transitions to the "off" state. Previous work had suggested the identification of the "off" state as an extreme manifestation of the weak state. However, the connection between the "off" and bright emission reported here suggests that the emission can be interpreted as undergoing only two emission states: a "bursting" state consisting of both bright pulses and nulls, and the weak emission state.
\end{abstract}

Unified Astronomy Thesaurus concepts: Radio pulsars (1353); Observational astronomy (1145); Astronomy data analysis (1858)

\section{Introduction}

PSR J1107-5907 is an isolated radio pulsar that was discovered in the Parkes $20 \mathrm{~cm}$ Multibeam Pulsar Survey of the Galactic plane (Lorimer et al. 2013). Its rotational period $(P \sim 0.25 \mathrm{~s})$ is typical of normal pulsars, but a comparatively low period derivative $\left(\dot{P} \sim 9 \times 10^{-18}\right)$ places this pulsar in an underpopulated region in the $P-\dot{P}$ diagram between the populations of normal and recycled pulsars. The inferred characteristic age of the pulsar $\left(\tau_{c} \sim 447 \mathrm{Myr}\right)$ indicates that it is among the oldest non-recycled pulsars.

Pulsar emission is known to be complex. Individual pulses vary in shape, phase, and intensity. At least one-third of pulsars exhibit the phenomenon known as subpulse drifting (Weltevrede et al. 2006), in which individual pulse components drift in pulse phase. Many pulsars also exhibit "nulling" (Backer 1970), during which the pulsed emission seemingly switches off for a few pulses at a time. Pulsars are termed "intermittent" if their emission ceases for long periods corresponding to a large number of missing pulses (for instance, the emission may cease for hours to years). ${ }^{15}$ Kramer et al. (2006), Camilo et al. (2012), and Lyne et al. (2017) have shown that the spin-down rate for intermittent pulsars decreases when the pulse emission is off.

\footnotetext{
15 The term "intermittent" is not well defined. Some authors only use this term for pulsars whose emission ceases for timescales measured in days. Other authors use the term also for pulsars whose emission ceases on a much longer timescale than the pulse period, for instance over minutes to hours.
}

Other pulsars show discrete emission states in which the emission does not completely switch off in either state. This is known as "mode-changing." Various authors (e.g., Wang et al. 2007; Lyne et al. 2010) have suggested that mode-changing and nulling are related phenomena. Pulsars with complex combinations of these emission phenomena have also been reported (e.g., Bhattacharyya et al. 2010; Zhang et al. 2019).

Some pulsars, including PSRs J1752+2359 (Lewandowski et al. 2004), J1938+2213 (Lorimer et al. 2013), and B0611+22 (Seymour et al. 2014), exhibit "bursting" emission, during which a large number of bright individual pulses are detected over a relatively short time interval. There is still no clear physical model for why this occurs.

As we argue in this work, PSR J1107-5907 exhibits a number of these emission features, including bursts. O'Brien et al. (2006) reported that the pulsed emission of PSR J1107 -5907 switches between a null state with no detectable integrated pulse profile, a weak mode with a narrow pulse, and a bright mode with a very broad pulse profile. In contrast, Young et al. (2014) argued that the pulsar only exhibits two emission states, strong and weak, with the previously proposed "off" state simply being an extreme end of the weak emission state. They also showed that the pulsar is likely a near-aligned rotator and that it does not exhibit any measurable variation in spin-down rate between the emission states. Hobbs et al. (2016) detected the pulsar using the Australian Square Kilometre Array Pathfinder (ASKAP) (with some contemporaneous 
Table 1

Observations that Contain the Bright Emission State for PSR J1107-5907

\begin{tabular}{|c|c|c|c|c|c|c|c|c|c|}
\hline \# & File Name & MJD & Project ID & Receiver & $\begin{array}{c}\text { Central Obs. Frequency } \\
(\mathrm{MHz})\end{array}$ & $\begin{array}{l}\text { Bandwidth } \\
\text { (MHz) }\end{array}$ & Backend & $\begin{array}{l}\text { Duration } \\
\quad(s)\end{array}$ & Obs. Mode \\
\hline 1 & p120811_231320.rf & 56150.9 & P574 & MULTI & 1382 & 400 & CASPSR & 2712 & fold \\
\hline 2 & s121018_213312.rf & 56218.8 & P832 & MULTI & 1369 & 256 & PDFB3 & 21479 & fold \\
\hline 3 & s121019_182134.rf & 56219.7 & $\mathrm{P} 456$ & MULTI & 1369 & 256 & PDFB3 & 17879 & fold \\
\hline 4 & s140714_095334_3.sf & 56852.4 & P863 & $1050 \mathrm{CM}$ & 732 & 64 & PDFB3 & 900 & search \\
\hline 5 & t140714_095333_3.sf & 56852.4 & P863 & $1050 \mathrm{CM}$ & 3094 & 1024 & PDFB3 & 900 & search \\
\hline 6 & t141023_222022.sf & 56953.9 & P863 & $1050 \mathrm{CM}$ & 3094 & 1024 & PDFB4 & 417 & search \\
\hline 7 & s141023_222023.sf & 56953.9 & P863 & $1050 \mathrm{CM}$ & 732 & 64 & PDFB3 & 417 & search \\
\hline 8 & t160510_124308_1.sf & 57518.5 & P863 & $1050 \mathrm{CM}$ & 3094 & 1024 & PDFB4 & 900 & search \\
\hline 10 & p160911_021816.rf & 57642.0 & P863 & $\mathrm{H}-\mathrm{OH}$ & 1382 & 400 & CASPSR & 3616 & fold \\
\hline 11 & t180123_02181.sf & 58141.6 & P863 & MULTI & 1369 & 256 & PDFB4 & 900 & search \\
\hline 12 & t180603_040025.rf & 58272.1 & PX500 & MULTI & 1369 & 256 & PDFB4 & 7199 & fold \\
\hline
\end{tabular}

Parkes observations) and found that the typical timescale between strong emission states is $\sim 3.7 \mathrm{hr}$ and that the duration of the bright state was typically a few minutes, but in one case it lasted for almost 40 minutes. Meyers et al. (2018) presented the first low-frequency detection of the pulsar with the Murchison Widefield Array at $154 \mathrm{MHz}$ and the simultaneous detection from the upgraded Molonglo Observatory Synthesis Telescope at $835 \mathrm{MHz}$. They found that the pulsar exhibits steep spectral indices for both the bright main pulse component and the precursor component, and the pulse energy distribution of the bright state is best parameterized by a log-normal distribution at both frequencies.

During the past few years this pulsar has been semi-regularly observed using the $64 \mathrm{~m}$ Parkes telescope. Most of these observations are short, quick-look observations carried out as part of the P595 PULSE@Parkes outreach project (e.g., Hobbs et al. 2009), interspersed with relatively long ( $\sim 1 \mathrm{hr}$ ) observations obtained for a project studying pulsar intermittency (with Parkes observing code P863) and long rise-to-set observations carried out during time assigned to and supported by the Commensal Radio Astronomy FAST Survey program (Li et al. 2018; with observing code PX500). The data sets comprise a number of observing modes and frequency bands and include both data folded at the known pulse period and "search-mode" data in which single pulses can be analyzed.

With an aim of assessing the identification of PSR J1107 -5907 as a bursting pulsar, we concentrate on the Parkes data sets that exhibit the strong bright state. In Section 2, we describe the observations and processing method. Our primary results are presented in Section 3, in which we study the occurrences of emission-state switches and also study the single-pulse energetics to support our hypothesis. We compare models of the emission states in Section 4 and conclude in Section 5 .

\section{Observations}

The observations described here were obtained with the Parkes $64 \mathrm{~m}$ radio telescope. All these observations are archived in the CSIRO data archive (Hobbs et al. 2011; data. csiro.au). Most are now publicly available, but those acquired within the last 18 months are embargoed. We visually inspected all the available observations of PSR J1107-5907 and selected data files in which the pulsar was detected in its bright state, exhibiting a very broad and unambiguously bright integrated profile. Table 1 lists, for each observation, the corresponding file name, the modified Julian date (MJD) of the observation start, project ID, observing frequency, observing mode (fold or search), backend instrument(s) used, and the observation length.

Some observations were obtained simultaneously in the 10 and $40 \mathrm{~cm}$ observing bands with the dual-band receiver, but the majority of the observations were in the $20 \mathrm{~cm}$ observing band using the central beam of the 13-beam multibeam receiver. A few were recorded using the $\mathrm{H}-\mathrm{OH}$ single-pixel receiver. A number of backend systems have been used for recording the data, including the Parkes Digital Filterbanks (PDFB3 and PDFB4) and the CASPER Parkes Swinburne Recorder (CASPSR). Detailed descriptions of the receivers and backend systems can be found in Manchester et al. (2013) and Young et al. (2014). The PDFB and CASPSR backend systems can be calibrated (calibration of both flux density and polarization) if (as is usually the case) a switched calibration signal was recorded either prior to or after the observation. The final flux calibration is carried out by relating the calibration signal to the known flux density of Hydra A using the PSRCHIVE pulsar signal processing system (Hotan et al. 2004; Manchester et al. 2013; Xie et al. 2019). We formed analytic templates for the bright and weak emission modes separately from our observations using PAAS and then estimated the flux density using PSRFLUX, which matches the template with the observation and determines the area under the template.

Search-mode data are folded to the pulse period or $30 \mathrm{~s}$ subintegrations using the DSPSR software package (van Straten \& Bailes 2011). The search-mode calibration files are folded at the calibration pulse period $(11.123 \mathrm{~Hz})$. We mitigated aliased signals and narrowband radio-frequency interference by excising channels within $5 \%$ of the band edge and those with a level substantially above a median-smoothed bandpass, respectively. The PSRCHIVE program PAC is used to perform flux (based on observations of Hydra A) and polarimetric calibration.

\section{Results}

In Figure 1 we show three representative examples of the flux density as a function of time for PSR J1107-5907. Panels (A), (B), and (C) correspond to observations 12, 9 and 10 in Table 1 respectively. (Note that Table 1 only lists the data files in which the pulsar was detected its bright state. The duration of the whole of observations 10 and 12 is longer that listed in Table 1.) The short bright states have flux densities around $\sim 100 \mathrm{mJy}$. Note that the flux density is plotted on a logarithmic scale and so where the measured flux density value is 


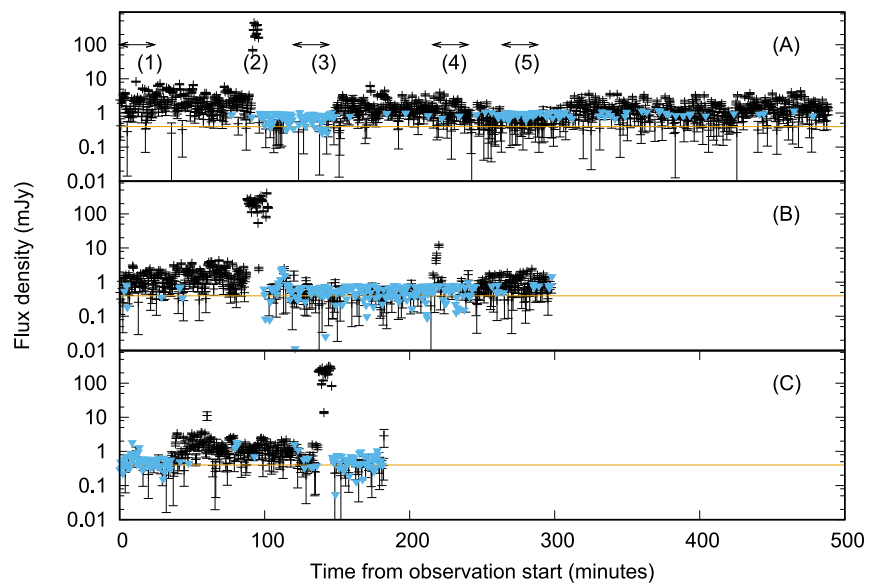

Figure 1. The variations in flux density of PSR J1107-5907 for observations including the bright phase. The flux density is plotted on a logarithmic scale. The yellow line indicates a flux density of $0.4 \mathrm{mJy} .3 \sigma$ upper bounds on the flux density are shown where no pulse is detectable as blue triangles.

consistent with zero we plot an upper bound symbol (downward-pointing triangle) at the value of the $1 \sigma$ uncertainty.

For the longest observation (Figure 1(A)) we identify five regions (labeled (1)-(5)). All the regions apart from region 2 are of 24 minutes duration. The folded, total intensity profiles for these regions are shown in Figure 2. Note that the $y$-axes use different scales for each panel. The pulsar is much more polarized during the bright mode than in the weak mode, and the linear and circular polarized profiles are similar in the three observing bands. As shown in Figure 3, there are three main linear polarized components in the main pulse, and the trailing component seems more linearly polarized than the precursor. As shown in Figures 1 and 2, the mean flux density during the bright state is about two orders of magnitude higher than that in the weak state. We have checked through all the calibrated data sets, and the mean flux density in the weak state fluctuates within an order of magnitude in all of the three observing bands, ranging from $0.7-4.2 \mathrm{mJy}, \quad 0.21-2.1 \mathrm{mJy}$, and $0.07-0.54 \mathrm{mJy}$ in the 10,20 , and $40 \mathrm{~cm}$ observing bands, respectively.

The pulsar flux density shown in region (1) of Figure 1(A) is around $2 \mathrm{mJy}$ at the start of the observation. This is typical of the weak state that has been described by Young et al. (2014) and O'Brien et al. (2006). There is a sudden change to the bright state (region (2)). The duration of the detected bright states ranges from a few minutes to a few tens of minutes and the intensity of strong single pulses during bright states can be higher than $10 \mathrm{Jy}$ (as shown in Figure 4) at $0.7 \mathrm{GHz}$. All the integrated profiles during bright states are similar but not identical since the strength and the number of single pulses during each bright state are different. After the bright state in panel (A) the pulsar seems to switch off. In region (3) we have no significant measurements of a pulse with an upper limit of $0.2 \mathrm{mJy}$. The folded pulse profile (Figure 2) shows no indication of a pulse. We explain below (Section 4) that this is a likely an indication of a bursting-emission state, and not, as Young et al. (2014) suggest, part of the weak emission state. Region (4) is back to the weak state (although the mean flux density of $1.2 \mathrm{mJy}$ is slightly lower than the mean value prior to the bright emission). Between regions (4) and (5) we identify a significant decrease in the flux density. However, in contrast to region (3), the emission in region (5) is still present (as seen in

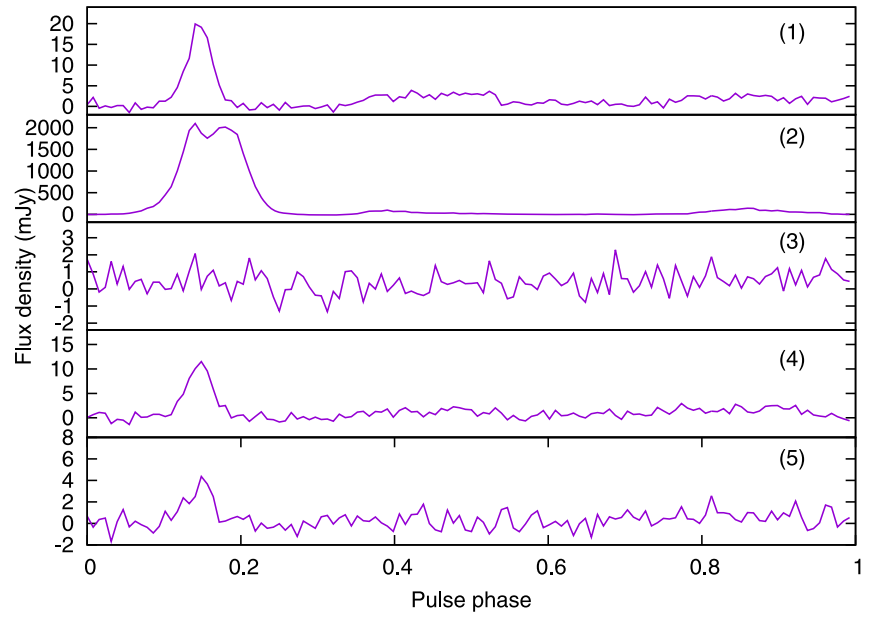

Figure 2. The integrated pulse profiles from the five different observation segments marked in Figure 1.

the bottom panel of Figure 2) and the mean flux density is 0.4 mJy. This is the so-called low-level "underlying emission" identified by Young et al. (2014), which is only detected through profile integration and commonly seen in our data set. The emission then increases back to the level of a typical weak state.

For this observation (panel (A)) the emission pattern is from weak at the start of the observation to bright, to off, and then to weak (though the final weak state varies significantly in flux density until the end of the observation). For all of the observations listed in Table 1 we have determined whether the emission transitions from "weak to off," "bright to off," "bright to weak," "off to weak," or "off to bright." These results are presented in Table 2. In all observed cases the emission switches from "bright to off," and never from the bright to the weak state. The majority of the observations exhibit the pattern "weak to bright to off," but in a few cases (observation numbers 7,10 , and 11 in Table 1) we observe "off to bright" and then back to off.

A more detailed look at an "off to bright to off" transition can be seen in Figure 4. In this figure we have shown the flux density of individual pulses at $1.4 \mathrm{GHz}$ around a bright emission state. For the first $\sim 7$ minutes we see no evidence of any emission. Then the pulsar abruptly enters the bright emission state. The bright state lasts for $\sim 5$ minutes and then the emission switches back off. However, we notice two individual, single pulses occurring just after 15 minutes from the start of the observation. These pulses are clearly from the pulsar (the inset shows the dispersion of the pulse). Based on the single-pulse observations listed in Table 1, we have determined that the pulsar seems always to enter the bright emission state suddenly, within one rotation of the neutron star.

We have two observations in which simultaneous dual-band $(10 / 40 \mathrm{~cm})$ single-pulse data sets were recorded while the pulsar was in the bright state (observation numbers 4, 5, 6, and 7 in Table 1). The variations in calibrated single-pulse flux densities are shown in Figure 5 for observation numbers 6 and 7 (note that observation files 6 and 7 represent DFB3 and DFB4 simultaneous observations of the pulsar in different observing bands). In order to compare the flux density in the two bands, the flux densities in the $10 \mathrm{~cm}$ observing band are shown as negative values. The flux densities for the sporadic single pulses seen during the weak state are at the level of a few 

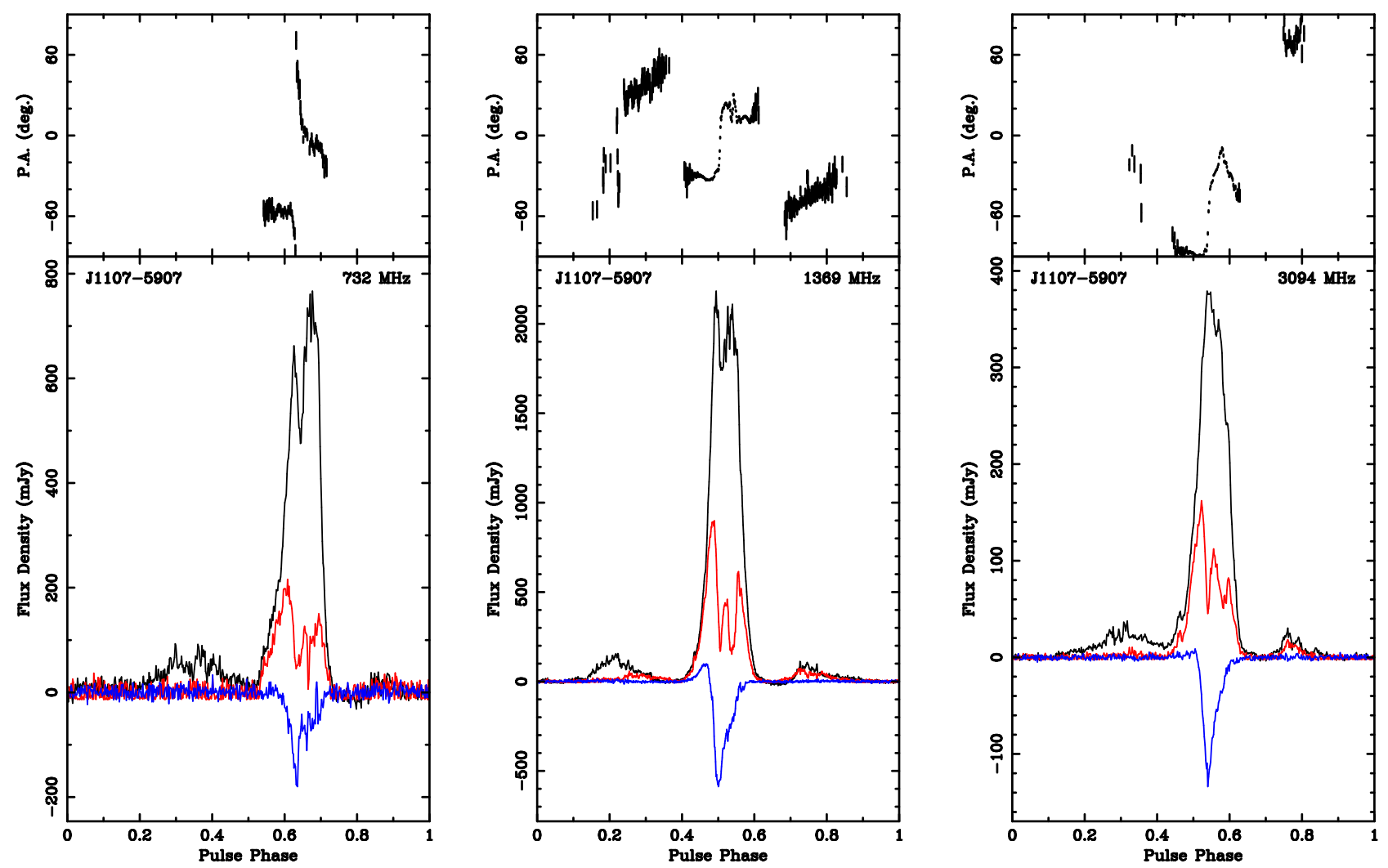

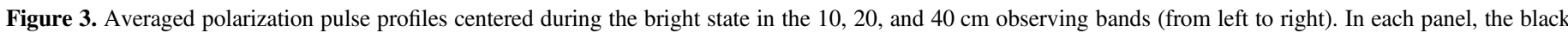
line shows total intensity, red linear polarization, and blue circular polarization. The position angle of the linear polarization is shown in the top panels.

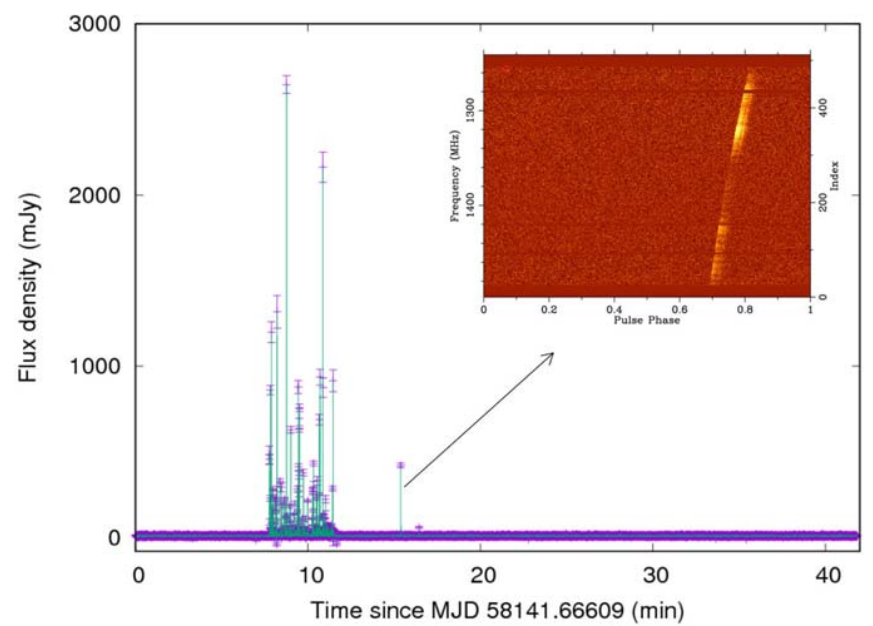

Figure 4. Calibrated fluxes of single pulses vs. time during state transitions from "off" to "bright" to "off." The inset shows frequency vs. phase for the strong single pulse that occurred after the bright state.

tens of mJy, one hundred mJy, and a few hundred mJy in the 10,20 , and $40 \mathrm{~cm}$ bands, respectively. They are more than an order of magnitude weaker than the strong single pulses in the bright state as shown in Figures 4 and 5.

These 453 single pulses show that the emission abruptly starts and stops, but it is "bursting"; the individual pulses vary significantly in terms of their flux densities and spectral index. We identify 28 pulses in which we clearly detect the pulse in the $40 \mathrm{~cm}$ observing band but not in the $10 \mathrm{~cm}$ band. and 40 pulses in which the pulse is only observed in the highfrequency band. We calculated the spectral index from 60
Table 2

Occurrences of the Various Combinations of the Possible State Switches for the 12 Observations that Contain the Bright State

\begin{tabular}{lc}
\hline \hline Transition & Occurrences \\
\hline weak to off & 0 \\
weak to bright & 9 \\
bright to off & 12 \\
bright to weak & 0 \\
off to weak & 4 \\
off to bright & 3 \\
\hline off to bright to off & 3 \\
off to bright to weak & 0 \\
weak to bright to off & 9 \\
weak to bright to weak & 0 \\
\hline
\end{tabular}

individually matched pulses (above a signal-to-noise threshold of 6 for both bands). The spectral index ranges from -3.56 to 1.37 with a mean of -1.11 and a standard deviation of 1.1 . The variation of the spectral index is more extreme than that presented by Meyers et al. (2018).

Characterizing the pulse energy distribution of a pulsar is helpful in understanding the pulse emission process. The pulse energy distribution of pulsars can often be represented by a single-component distributions (a log-normal or a power-law distribution). Since our data are well calibrated, we identify the pulse energy with the flux density. Below, we fit the pulse energy distribution for the bright states of observation numbers 6 and 11 in Table 1 (see also Figure 4 and the red line in Figure 5) and display the resulting best-fit model in Figure 6. 


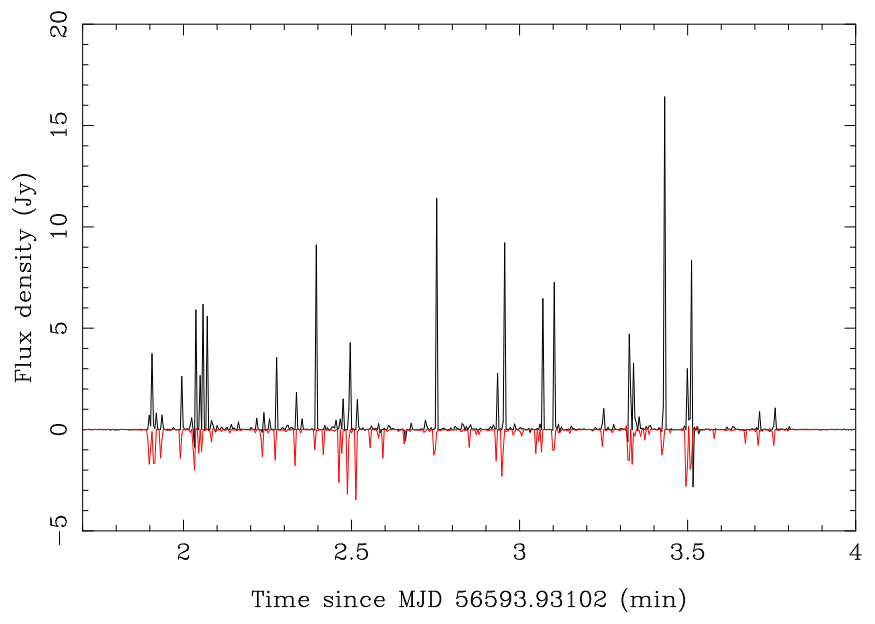

Figure 5. The variation in dual-band $(10 / 40 \mathrm{~cm})$ single-pulse flux density during a bright states. The $40 \mathrm{~cm}$ data are shown as positive flux densities in black and the $10 \mathrm{~cm}$ data as negative values in red.

We modeled the pulse energy distribution with both a lognormal and a power-law distribution, and we also considered an additional null component for each case. In detail, we first characterized the white noise properties of the pulse distribution, finding it to be well described by a normal distribution, and then convolved the trial distribution (log-normal or powerlaw) with the white noise model to determine the predicted distribution of pulse intensities. We determined the parameters of the distribution by optimizing the Gaussian likelihood. The specific form of the power law, $P(F) \propto\left(1+\left(F / F_{c}\right)^{2}\right)^{\alpha / 2}$, includes a low-energy cutoff, $F_{c}$, in addition to the spectral index $\alpha$. The log-normal distribution follows the standard form $P(F) \propto(1 / \sigma F) \exp \left[-0.5(\ln F-\mu)^{2} / \sigma^{2}\right]$ with parameters for the logarithmic width $(\sigma)$ and mean $(\mu)$ of the distribution. We ignored a small number of negative outliers that were formally below $4 \sigma$ for our white noise distribution, as well as the long region of nulls shown in Figure 4. We incorporate nulls using a mixture model whose relative normalization (nulling fraction) is the only additional free parameter.

After optimizing the parameters for both models, we find that the power-law distribution is preferred by an increase in the log-likelihood of $\sim 4$ for each of the two data sets. While this increase is formally significant, given the potential influence of systematic errors in the flux densities, we argue that both models provide an adequate description of the pulse energy distribution. We also considered the presence of an additional component of nulls, interleaved with the pulse energies described by the power-law and log-normal distributions. With this component, the log-likelihood improves by $\sim 4$ for the log-normal model for both sets, and by 18 and 13 for the power law. A null component is, formally, strongly preferred for the power-law model, though we again caution that systematic flux errors could dilute this preference. The bestfit nulling fraction is $25 \%$ and $30 \%$ for the two data sets for the log-normal model, and $35 \%$ and $45 \%$ for the power-law model. (We again emphasize that we have excluded the obvious long span of nulls for observation number 11 in Table 1.) In summary, there is no strong preference for either model, and only modest evidence for nulling under the log-normal description. However, if the power-law model is the correct underlying distribution, there is good evidence for a nulling fraction of $35 \%-45 \%$.

\section{Discussion}

We believe that there are three possible descriptions of the emission states in this pulsar.

1. As originally described by O'Brien et al. (2006), the pulsar exhibits three emission states: weak, strong, and off.

2. As proposed by Young et al. (2014), there are only two emission states: weak and strong. Here, the "off" state is only the weakest part of the weak state.

3. A new description in which there are only two states, which we label "persistent" and "bursting." The persistent state is the same as the "weak" state described earlier. However, the "bursting" state contains very bright single pulses (leading to the bright emission), but also long periods of nulls (leading to the off state).

We note that more sensitive telescopes may be able to detect emission from the off state. Our current observational results are inconsistent with description \#2 because there seems to be a repeating pattern of "bright" to "off," which is hard to explain if the off state is simply a part of the weak state with a low signal-to-noise ratio $(\mathrm{S} / \mathrm{N})$.

Distinguishing between descriptions \#1 and \#3 is difficult. But a model with nulls occurring beside bright pulses is mildly preferred. We have tried to measure the pulse frequency and its derivative during different states. However, the measured precision of these quantities is insufficient to distinguish them. The number of nulls between two bright single pulse in the bright state ranges from 1 to $\sim 1200$ (as shown in Figure 4 . The bright state often ends with many nulls as shown in Figure 4, but the number of nulls after the bright state varies widely between observations. During the weak state, many detectable single pulses can often be seen and they form the visible integrated pulse profile. The flux density of these detectable single pulses is at the level of $\sim 100 \mathrm{mJy}$ at $1.4 \mathrm{GHz}$ and a few tens of mJy at $3.0 \mathrm{GHz}$, which is much weaker than single pulses that occur during the bright state. As shown in Figure 1, the flux densities fluctuate rapidly over a small range during the weak state. Scintillation structure is clearly visible in the single dispersed pulse from the inset of Figure 4, raising the possibility that interstellar scintillation could influence the inferred properties of the single pulses. We estimate a scintillation bandwidth of $73 \mathrm{MHz}$ from a frequency-domain autocorrelation analysis. It is more difficult to estimate the scintillation timescale since the pulsar is in the weak state for the most of the time; however, no obvious change in the scintillation structure is observed during the longest bright state, setting a lower limit on the diffractive timescale of 24 minutes. We can thus conclude that the inferred properties of a single pulse are not affected by interstellar scintillation.

Pulsar state switching behaviors including nulling, bursting, mode changes, subpulse drifts, and long-term intermittency can all be modeled as Markov processes (Cordes 2013). Kerr et al. (2014) modeled the nulling of PSR J1717-4054 as a threestate Markov process. However, in order to model the state switching of the pulsar in this paper, the different states need to be well defined via the single-pulse properties first. Quantitative evaluation of a Markov model also requires observations with sufficient $\mathrm{S} / \mathrm{N}$.

Even for many well-studied objects, pulse-to-pulse variations cause overlap of on- and off-state intensities, leading to false positives from algorithms that identify state changes. 

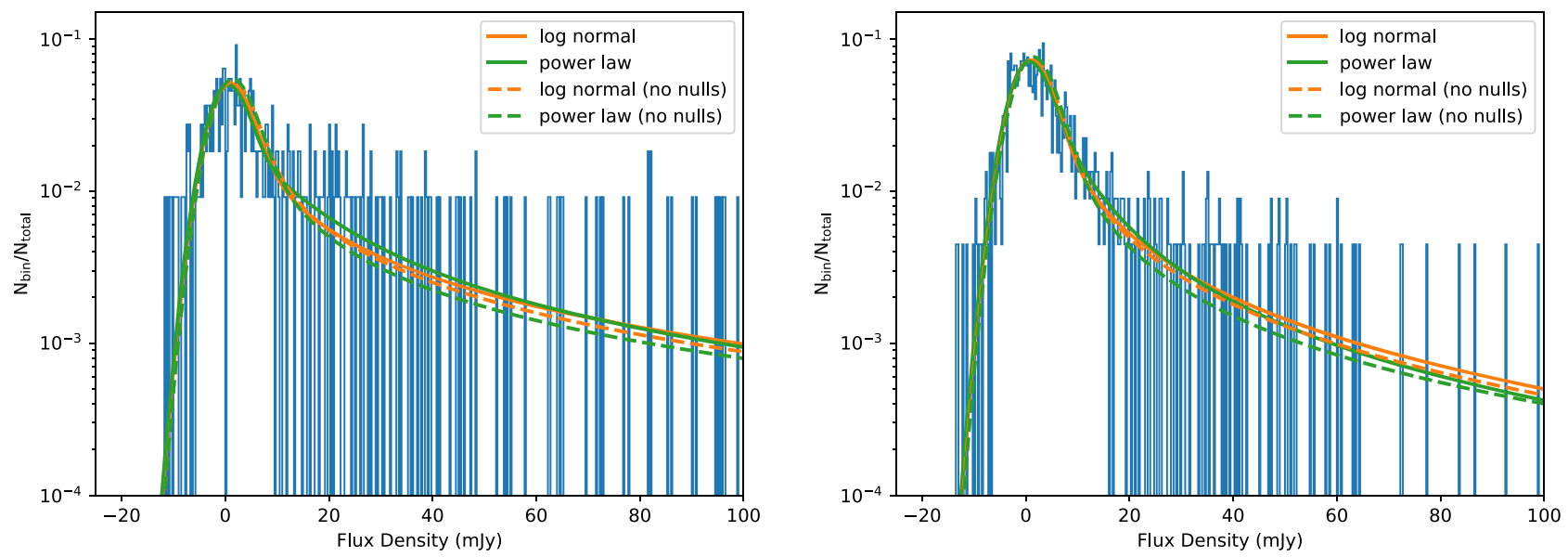

Figure 6. The pulse energy distributions and best-fit models during the bright state for observation numbers 6 (left) and 11 (right) in Table 1.

Future work with now-available wideband receiver systems on existing telescopes and eventually with new array telescopes (ASKAP, MeerKAT, and the Square Kilometre Array (SKA)) can improve the discrimination between states as well as expand the sample of objects that can be studied in this way.

\section{Conclusion}

Our analysis of PSR J1107-5907 observations is not consistent with the model of Young et al. (2014) in which the pulsar exhibits only a bright and a weak emission state. However, our results are consistent with a two-state model in which the pulsar is in a persistent state or in a bursting-emission state.

Continued monitoring of this pulsar is essential to understand the emission in more detail, but there are two clear areas that could be improved from the current Parkes observations. First, a much more sensitive telescope could probe the pulse nulls/very weak/off state in more detail and then different states of the pulsar can be well defined and used as input for a Markov model of the state switching process. PSR J1107 -5907 is in the far southern sky and so, until the SKA era, the only telescope capable of providing more sensitive observations is MeerKAT, which is about seven times more sensitive than Parkes multibeam observations. Second, the dual-band $10 / 40 \mathrm{~cm}$ data indicate significant variations in the single-pulse properties across that band. The recently installed ultrawidebandwidth receiver at Parkes provides uninterrupted coverage over the entire band from $800-4000 \mathrm{MHz}$ with greater sensitivity than currently possible.

The Parkes radio telescope is part of the Australia Telescope, which is funded by the Commonwealth of Australia for operation as a National Facility managed by the Commonwealth Scientific and Industrial Research Organisation (CSIRO). This paper includes archived data obtained through the CSIRO Data Access Portal (http://data.csiro.au). This work is supported by the Youth Innovation Promotion Association of Chinese Academy of Sciences, 201* Project of Xinjiang Uygur Autonomous Region of China for Flexibly Fetching in Upscale Talents, the National Key R\&D Program of China (No. 2017YFA0402602), the National Natural Science Foundation of China (No. 11690024), and the Strategic Priority Research Program (B) of the Chinese Academy of Sciences (No. XDB230102000). R.S. acknowledge Australian Research Council grant FL150100148. Work at NRL is supported by NASA.

\section{ORCID iDs}

Jingbo Wang (iD https://orcid.org/0000-0001-9782-1603 Matthew Kerr (iD https://orcid.org/0000-0002-0893-4073 Shi Dai (ib https://orcid.org/0000-0002-9618-2499 Vikram Ravi (i) https://orcid.org/0000-0002-7252-5485

\section{References}

Backer, D. C. 1970, Natur, 228, 42

Bhattacharyya, B., Gupta, Y., \& Gil, J. 2010, MNRAS, 408, 407

Camilo, F., Ransom, S. M., Chatterjee, S., Johnston, S., \& Demorest, P. 2012, ApJ, 746, 63

Cordes, J. M. 2013, ApJ, 775, 47

Hobbs, G., Heywood, I., Bell, M. E., et al. 2016, MNRAS, 456, 3948

Hobbs, G., Hollow, R., Champion, D., et al. 2009, PASA, 26, 468

Hobbs, G., Miller, D., Manchester, R. N., et al. 2011, PASA, 28, 202

Hotan, A. W., van Straten, W., \& Manchester, R. N. 2004, PASA, 21, 302

Kerr, M., Hobbs, G., Shannon, R. M., et al. 2014, MNRAS, 445, 320

Kramer, M., Lyne, A. G., O’Brien, J. T., et al. 2006, Sci, 312, 549

Lewandowski, W., Wolszczan, A., Feiler, G., Konacki, M., \& Sołtysiński, T. 2004, ApJ, 600, 905

Li, D., Wang, P., Qian, L., et al. 2018, IMMag, 19, 112

Lorimer, D. R., Camilo, F., \& McLaughlin, M. A. 2013, MNRAS, 434, 347

Lyne, A., Hobbs, G., Kramer, M., et al. 2010, Sci, 329, 408

Lyne, A. G., Stappers, B. W., Freire, P. C. C., et al. 2017, ApJ, 834, 72

Manchester, R. N., Hobbs, G., Bailes, M., et al. 2013, PASA, 30, e017

Meyers, B. W., Tremblay, S. E., Bhat, N. D. R., et al. 2018, ApJ, 869, 134

O’Brien, J. T., Kramer, M., Lyne, A. G., Lorimer, D. R., \& Jordan, C. A. 2006, ChJAS, 6, 4

Seymour, A. D., Lorimer, D. R., \& Ridley, J. P. 2014, MNRAS, 439, 3951 van Straten, W., \& Bailes, M. 2011, PASA, 28, 1

Wang, N., Manchester, R. N., \& Johnston, S. 2007, MNRAS, 377, 1383 Weltevrede, P., Edwards, R. T., \& Stappers, B. W. 2006, A\&A, 445, 243 Xie, Y.-W., Wang, J.-B., Hobbs, G., et al. 2019, RAA, 19, 103

Young, N. J., Weltevrede, P., Stappers, B. W., Lyne, A. G., \& Kramer, M. 2014, MNRAS, 442, 2519

Zhang, L., Li, D., Hobbs, G., et al. 2019, ApJ, 877, 55 